\title{
Comparison of clinical utility between diaphragm excursion and thickening change using ultrasonography to predict extubation success
}

Jung-Wan $\mathrm{Yoo}^{1,}{ }^{1,}$, Seung Jun Lee ${ }^{2}$, Jong Deog Lee ${ }^{2}$, and Ho Cheol Kim²

${ }^{1}$ Division of Pulmonary and Critical Care Medicine, Department of Medicine, Samsung Changwon Hospital, Sungkyunkwan University School of Medicine, Changwon; ${ }^{2}$ Division of Pulmonary and Critical Care Medicine, Department of Internal Medicine, Gyeongsang National University Hospital, Jinju, Korea

Received: May 12, 2016 Revised : August 3, 2016 Accepted: October 13, 2016

\section{Correspondence to} Ho Cheol Kim, M.D.

Department of Internal Medicine, Gyeongsang National University Hospital, 79 Gangnam-ro,

Jinju 52727, Korea

Tel: $+82-55-750-8684$

Fax: +82-55-750-8618

E-mail: hochkim@gnu.ac.kr

*Current affiliation: Division of Pulmonary and Critical Care Medicine, Department of Internal Medicine, Gyeongsang National University Hospital, JinJu, Korea
Background/Aims: Both diaphragmatic excursion and change in muscle thickening are measured using ultrasonography (US) to assess diaphragm function and mechanical ventilation weaning outcomes. However, which parameter can better predict successful extubation remains to be determined. The aim of this study was to compare the clinical utility of these two diaphragmatic parameters to predict extubation success.

Methods: This study included patients subjected to extubation trial in the medical or surgical intensive care unit of a university-affiliated hospital from May 2015 through February 2016. Diaphragm excursion and percent of thickening change ( $\Delta$ tdi\%) were measured using US within 24 hours before extubation.

Results: Sixty patients were included, and 78.3\% (47/60) of these patients were successfully extubated, whereas $21.7 \%$ (13/60) were not. The median degree of excursion was greater in patients with extubation success than in those with extubation failure $(1.65 \mathrm{~cm}$ vs. $0.8 \mathrm{~cm}, p<0.001)$. Patients with extubation success had a greater $\Delta$ tdi\% than those with extubation failure ( $42.1 \%$ vs. $22.5 \%, p=0.03$ ). The areas under the receiver operating curve for excursion and $\Delta$ tdi\% were $0.836(95 \%$ confidence interval [CI], o.717 to 0.919$)$ and 0.698 ( $95 \% \mathrm{CI}, 0.566$ to 0.810 ), respectively $(p=0.017)$.

Conclusions: Diaphragm excursion seems more accurate than a change in the diaphragm thickness to predict extubation success.

Keywords: Extubation; Diaphragm; Ultrasonography; Excursion; Thickness

\section{INTRODUCTION}

The appropriate timing of extubation is crucial in critically ill patients who wean from invasive mechanical ventilation. Early extubation causes post-extubation respiratory failure that result in reintubation and poor outcomes [1]. Delayed extubation leads to endotracheal obstruction, tracheal stenosis or pneumonia, which contributes to poor prognosis. Therefore, deciding the appropriate time for extubation remains a challenge to intensivists who care for critically ill patients.

There are several parameters to decide to extubate, but these have still have limitations to achieve successful extubation [2]. The diaphragm is the primary muscle of inspiration used for spontaneous breathing, therefore the assessment of diaphragm dysfunction is pivotal in 
patients to be subjected to weaning or extubation trial [3]. Although variable methods to evaluate diaphragm function are available [4], ultrasonography (US) has emerged as a tool to predict weaning from mechanical ventilation or extubation in the intensive care unit (ICU) [5]. Diaphragm function can be assessed by two parameters, namely excursion and diaphragm thickness change during spontaneous breathing, which were associated with weaning or extubation $[6,7]$. However, these two parameters had not been compared with each other to determine which one can better predict successful extubation.

The aim of this study was to compare the clinical utility of two diaphragmatic parameters to predict extubation success.

\section{METHODS}

\section{Subject}

From May 2015 to February 2016, subjects who were prepared for weaning and extubation were enrolled in the 28-bed medical or surgical ICU at a tertiary hospital. The clinical data were collected and analyzed, retrospectively. Patients were mechanically ventilated for at least 48 hours before spontaneous breathing trial(SBT). Patients were included when they met all of the following criteria for the SBT: age $\geq 18$ years, $\mathrm{FiO}_{2}<50 \%$, positive end-expiratory pressure level $\leq 5 \mathrm{cmH} 2 \mathrm{O}$, respiratory rate $\leq 30$ breaths/min, $\mathrm{PaO} 2 / \mathrm{FiO}_{2}>200 \mathrm{mmHg}$, Glasgow coma score $\geq 14$, and hemodynamic stability in the absence of vasopressors. The exclusion criteria were as follows: pregnancy, age $<18$ years, and history of diaphragmatic palsy or neuromuscular disease.

Extubation was performed if all of the following criteria were satisfied during the 30 to 120 minute SBT: good tolerance to SBT, respiratory rate $<35$ breaths/minute, heart rate $<140$ beats/minute or heart rate variability $\leq 20 \%$, oxygen saturation $\geq 90 \%, 80 \mathrm{mmHg}<$ systolic blood pressure $<180 \mathrm{mmHg}$ or $<20 \%$ change from baseline and absence of increased breathing work or distress signs.

This study was approved by the Institutional Review Board (IRB) of ethics committee of Gyeongsang National University Hospital(IRB No.: GNUH 2015-10-015-003). Informed consent was waived owing to the retrospective feature of this study.

\section{Measurement of diaphragm excursion or thickness}

Weaning trials consist of SBT (t-piece) or pressure support (PS) trials (reducing PS to $5 \mathrm{cmH}_{2} \mathrm{O}$ with less than $5 \mathrm{cmH}_{2} \mathrm{O}$ positive end-expiratory pressure). Both excursion and thickness of diaphragm (tdi) were measured on the same patients during tidal breathing. The diaphragm function was evaluated at right hemidiaphragm. All measurements were conducted by the same examiner (J.W.Y). The diaphragm movement was measured with a 2 to $5 \mathrm{MHz}$ US probe (M-Turbo, Fujifilm SonoSite Inc., Bothell, WA, USA) placed over one of the lower intercostal spaces in the right anterior axillary lines for the right diaphragm and the liver serving as an acoustic window. A two-dimensional (2D) mode was used to search for the line of the right hemidiaphragm. An angle of more than $70^{\circ}$ between the hemidiaphragm dome and US beam was applied. In the M-mode, the diaphragmatic excursion (displacement, $\mathrm{cm}$ ) was identified and measured (Fig. 1A and 1D). The excursion amplitude was measured on the vertical axis tracing from the baseline to the point of maximum height of inspiration on the graph as previously described [5]. The tdi was measured using a 6 to $13 \mathrm{MHz}$ linear US probe set to $\mathrm{B}$ mode ( $\mathrm{M}$-Turbo). The right hemidiaphragm was imaged at the zone of apposition of the diaphragm and rib cage in the mid-axillary line between the 8th and 10th intercostal spaces as previously described [8]. The tdi was measured at the end-expiration and end-inspiration (Fig. $1 \mathrm{~B},{ }_{1} \mathrm{C},{ }_{1} \mathrm{E}$, and ${ }_{1} \mathrm{~F}$ ). The percent change in tdi $(\Delta$ tdi\%) between end-expiration and end-inspiration was calculated as follows (tdi end-inspiration-tdi end-expiration/tdi end-expiration) $\times 100[7]$.

\section{Definition of extubation success or failure}

Extubation success was defined as sustained spontaneous breathing for more than 48 hours following extubation without noninvasive ventilation. Extubation failure was defined as reintubation and application of noninvasive ventilation within 48 hours or requiring tracheostomy [9].

\section{Statistical analysis}

Data are presented as the median and interquartile range (IQR) for continuous variables and number (\%) 

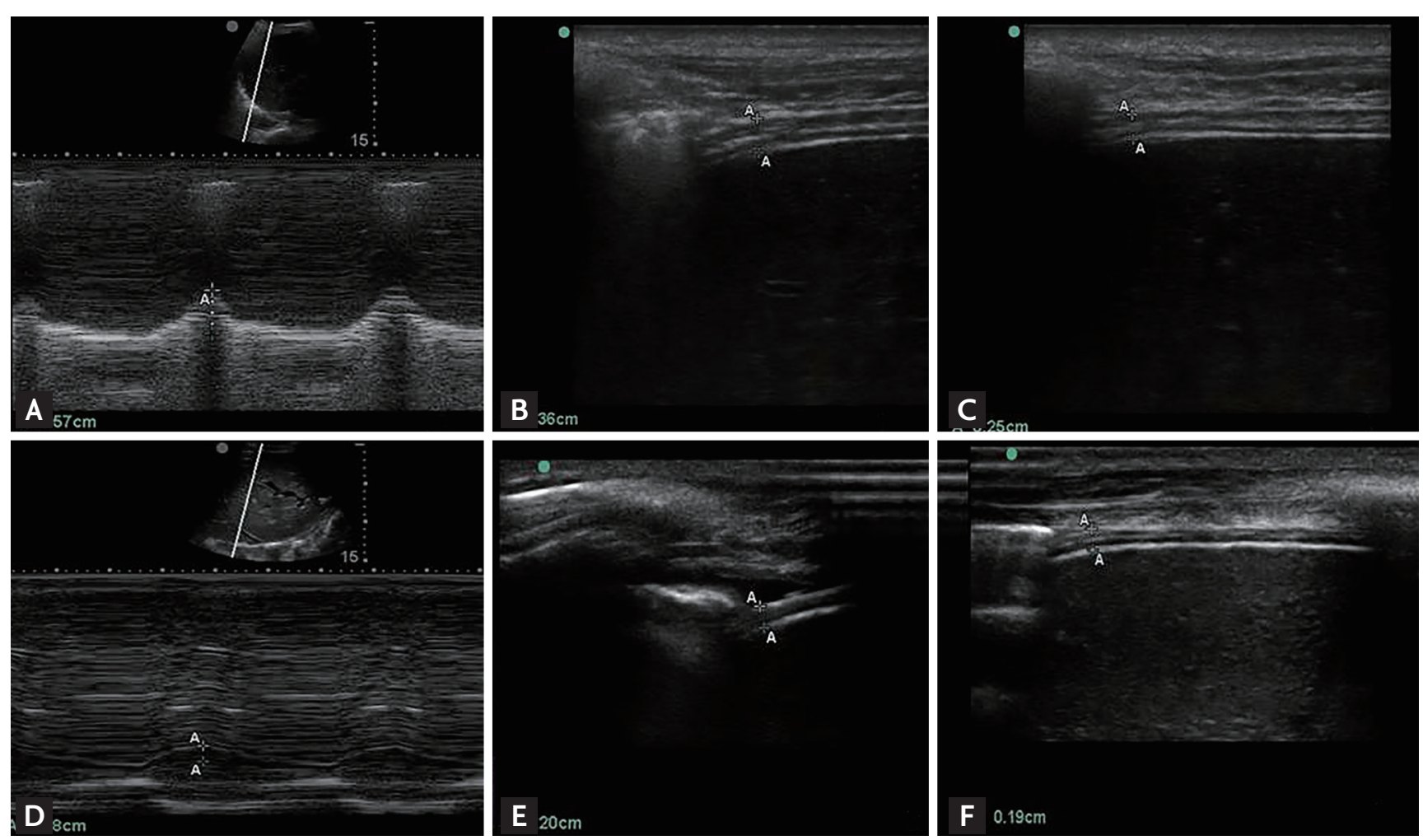

Figure 1. M-mode and B-mode of diaphragm excursion (A) and thickness (B, inspiration; C, expiration) in a patient with extubation success and M-mode and B-mode of excursion (D) and thickness (E, inspiration; F, expiration) in a patient with extubation failure.

for noncontinuous variables. Chi-square or Fisher exact tests were used to compare categorical variables and Mann-Whitney $U$ tests were used to compare continuous variables. Correlations were tested using spearman's method. The sensitivity, specificity, positive predictive value (PPV) and negative predictive value (NPV) were calculated for excursion and tdi to predict extubation success. The receiver operating characteristic (ROC) curves were used to evaluate and compare the clinical utility of the diaphragm excursion and ( $\Delta$ tdi\%) for extubation success. A $p$ value $<0.05$ was considered statistically significant. The data were analyzed using SPSS version 18.0 (SPSS Inc, Chicago, IL, USA) and MedCalc version 15.2.2 (MedCalc Software, Ostend, Belgium).

\section{RESULTS}

\section{Baseline characteristics and diaphragm parameters assessed via US}

Sixty patients were eligible during the study period. Fifty-five patients (91.7\%) were subjected to weaning trial with T-piece and five patients (8.3\%) had low PS ventilation. All patients were extubated within 24 hours of measuring diaphragm excursion and thickening change. The baseline characteristics are presented in Table 1. The median age was 69.5 years (IQR, 57.5 to 76.0) and approximately $70 \%$ of the patients were male. Fifty patients (83.3\%) were admitted to the medical ICU. About twothirds of the patients had hypoxemic respiratory failure. The median duration of invasive mechanical ventilation before the weaning trial was 5 days (IQR, 3 to 8.75).

The median diaphragm excursion was $1.53 \mathrm{~cm}$ (IQR, 0.89 to 1.9). The proportions of subjects with excursion degree more than 1 and $1.4 \mathrm{~cm}$ were $70 \%(\mathrm{n}=42)$ and $55 \%(\mathrm{n}=33)$, respectively. The median end-inspiration and end-expiration tdi were $0.29 \mathrm{~cm}$ (IQR, 0.22 to 0.35 ) and $0.2 \mathrm{~cm}$ (IQR, 0.17 to 0.24 ), respectively. The median $\Delta$ tdi\% between end-expiration and end-inspiration was $36.2 \%$ (IQR, 16.9 to 53.4 ). The proportions of $\Delta \mathrm{tdi} \geq 20 \%$ and $\geq 30 \%$ were $71.7 \%(n=43)$ and $61.7 \%(n=37)$, respectively. 


\section{Comparision of baseline and diaphragm parameters between patients with extubation success and failure}

The duration between the measurement of excursion or tdi and extubation was within 24 hours for all subjects; 39 (65\%) within 6 to 12 hours and 21 (35\%) within 12 to 24 hours. Among sixty patients, 47 (78.3\%) achieved extubation success, whereas 13 (21.7\%) did not. Table 2 summarizes the comparison of baseline and clinical characteristics between subjects with extubation success and those with failure. A trend toward longer duration of mechanical ventilation was shown in subjects with extubation failure than those with extubation success. Table 3 compares the physiologic variables and diaphragm

Table 1. Baseline characteristics of all patients $(n=60)$

\begin{tabular}{lc}
\hline Characteristic & No. (\%) \\
\hline Age, yr (IQR) & $69.5(57.5-76.0)$ \\
\hline Male sex & $42(70.0)$ \\
\hline Body mass index, $\mathrm{kg} / \mathrm{m}^{2}(\mathrm{IQR})$ & $21.8(19.1-24.9)$ \\
\hline Simplified Acute Physiology Score & $69.5(59.3-77.8)$ \\
III (IQR) & \\
\hline Comorbidity & $9(15.0)$ \\
\hline History of tuberculosis & $14(23.3)$ \\
\hline Diabetes mellitus & $9(15.0)$ \\
\hline Malignancy & $14(23.3)$ \\
\hline Chronic lung disease & $5(8.3)$ \\
\hline Chronic kidney disease & $2(3.3)$ \\
\hline Chronic heart disease & $10(16.7)$ \\
\hline Chronic liver disease & $9(15.0)$ \\
\hline Cerebrovascular disease & \\
\hline Drug affecting diaphragm dysfunction & $14(23.3)$ \\
\hline Neuromuscular blocking agents & $17(28.3)$ \\
\hline Corticosteroid & $3(5.0)$ \\
\hline Aminoglycoside & $11(18.3)$ \\
\hline Aminophylline & $2(3.3)$ \\
\hline Organophosphate & $37(61.7)$ \\
\hline Type of respiratory failure & $23(38.3)$ \\
\hline Hypoxemic & \\
\hline Hypercapnic & $35(58.3)$ \\
\hline Status upon intensive care unit & $7(11.7)$ \\
\hline admission & \\
\hline Acute sepsis or septic shock & \\
\hline & \\
\hline
\end{tabular}

IQR, interquartile range. measurements at the time of weaning trial between subjects with extubation success and those with extubation failure. No differences were observed in terms of physiologic variables between two groups. Subjects with extubation success had significantly greater excursion degree than those with extubation failure. Additionally, Subjects with extubation success had a significantly greater $\Delta$ tdi\% than those with extubation failure. The diaphragm excursion degree was positively, but moderately, correlated with $\Delta$ tdi\% $(r=0.450, p<0.001)$. The subgroup analysis of sonographic diaphragm parameters was performed based on the types of respiratory failure (Table 4). In hypercapnic respiratory failure, subjects with extubation success had significantly greater excursion degree and $\Delta$ tdi\% than those with extubation failure. In hypoxemic respiratory failure; however, no difference in excursion degree and $\Delta$ tdi\% was detected between the two groups.

Table 5 shows the sensitivity, specificity, PPV, and NPV of sonographic indexes for patients with extubation success. Fig. 2 depicts the ROC curves for excursion degree and $\Delta$ tdi\%. The areas under the ROC curve for excursion degree and $\Delta$ tdi\% were 0.836 (95\% confidence interval [CI], 0.717 to 0.919 ) and 0.698 (95\% CI, 0.566 to $0.810)$, respectively with significant difference noted $(p$ $=0.017$ )

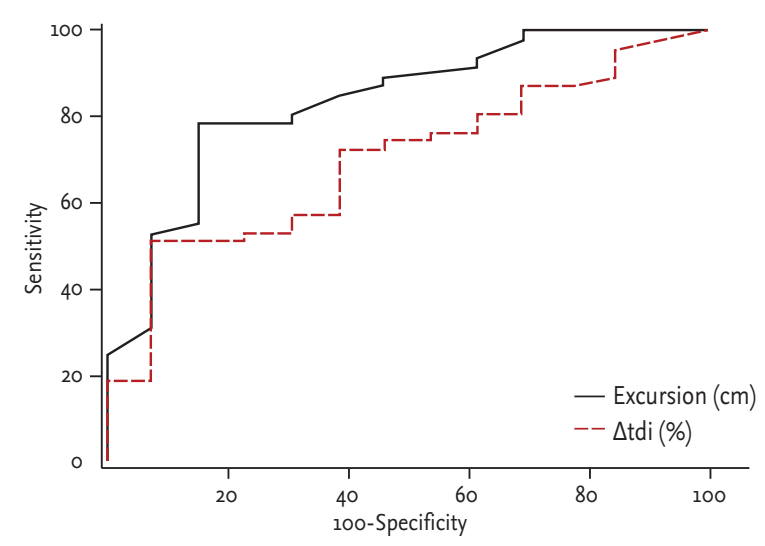

Figure 2. Comparison of receiver operating characteristic (ROC) for diaphragm excursion and $\Delta$ tdi\%. Area under the ROC curves for excursion: 0.836 (95\% CI, 0.717 to 0.919 ) and for $\Delta$ tdi\%: 0.698 (95\% CI, 0.566 to 0.810 ), $p=0.0177$. tdi, diaphragm thickness; $\Delta$ tdi\%, percent change of tdi between end-expiration and end-inspiration; CI, confidence interval. 


\section{Comparison of clinical outcomes between subjects with extubation success and those with extubation failure and causes of extubation failure}

Tracheostomy was performed in $46.2 \%$ (6/13) of patients who had extubation failure. The overall ICU mortality was $8.3 \%(5 / 55)$. Furthermore, 30.8\% (4/13) of patients with extubation failure died in the ICU compared with $2.1 \%(1 / 47)$ of those with extubation success $(p=0.0056)$. The median duration of ICU stay was 9.5 days (IQR, 5 to 15.3). Length of ICU stay was significantly longer in sub- jects with extubation success than those with extubation failure (7 days [IQR, 5 to 12] in extubation success vs. 12 days [IQR, 8 to 26$]$ in extubation failure; $p=0.015$ ). The possible causes of extubation failure in 13 patients were as follows: inability to remove respiratory secretion (five patients, $38.4 \%$ ), increased work of breathing, characterized by use of respiratory accessary muscle or paradoxical abdominal movement (six patients, $46.2 \%$ ), acute exacerbation of interstitial lung disease (one patient, $7.7 \%$ ), and hemodynamic instability caused by intraabdominal bleeding (one patient, 7.7\%).

Table 2. Comparison of baseline and clinical characteristics between patients with extubation success and those with extubation failure

\begin{tabular}{|c|c|c|c|}
\hline Variable & $\begin{array}{l}\text { Extubation success } \\
\qquad(\mathrm{n}=47)\end{array}$ & $\begin{array}{l}\text { Extubation failure } \\
\qquad(\mathrm{n}=13)\end{array}$ & $p$ value \\
\hline Age, yr (IQR) & $70(57-77)$ & $68(52-76)$ & 0.760 \\
\hline Male sex & $35(74.5)$ & $7(53.8)$ & 0.181 \\
\hline Body mass index, $\mathrm{kg} / \mathrm{m}^{2}$ (IQR) & $21.4(19.0-24.9)$ & $22.3(20.2-23.5)$ & 0.859 \\
\hline Simplified Acute Physiology Score III (IQR) & $71(63-77)$ & $59(51-81)$ & 0.190 \\
\hline \multicolumn{4}{|l|}{ Comorbidity } \\
\hline History of tuberculosis & $9(19.1)$ & 0 & 0.184 \\
\hline Diabetes mellitus & $12(25 \cdot 5)$ & $2(15 \cdot 4)$ & 0.713 \\
\hline Malignancy & $9(19.1)$ & 0 & 0.184 \\
\hline Chronic lung disease & $11(23 \cdot 4)$ & $3(23.1)$ & 1.000 \\
\hline Chronic heart disease & $1(2.1)$ & $1(7 \cdot 7)$ & 0.389 \\
\hline Chronic kidney disease & $5(10.6)$ & o & 0.575 \\
\hline Chronic liver disease & $7(14 \cdot 9)$ & $3(23.1)$ & 0.675 \\
\hline Cerebrovascular disease & $8(17.0)$ & $1(7 \cdot 7)$ & 0.668 \\
\hline \multicolumn{4}{|l|}{ Drugs affecting diaphragm dysfunction } \\
\hline Neuromuscular blocking agents & $12(25 \cdot 5)$ & $2(15 \cdot 4)$ & 0.713 \\
\hline Corticosteroid & $15(31.9)$ & $2(15 \cdot 4)$ & 0.314 \\
\hline Aminoglycoside & $2(4 \cdot 3)$ & $1(7 \cdot 7)$ & 0.526 \\
\hline Aminophylline & $9(19.1)$ & $2(15 \cdot 4)$ & 1.000 \\
\hline Organophosphate & 0 & $2(15 \cdot 4)$ & 0.044 \\
\hline Types of respiratory failure & & & 0.063 \\
\hline Hypoxemic respiratory failure & $32(68.1)$ & $5(38.5)$ & \\
\hline Hypercapnic respiratory failure & $15(31.9)$ & $8(61.5)$ & \\
\hline \multicolumn{4}{|l|}{ Status upon intensive care unit admission } \\
\hline Severe sepsis or septic shock & $30(63.8)$ & $5(38.5)$ & 0.122 \\
\hline Acute respiratory distress syndrome & $7(14.9)$ & o & 0.329 \\
\hline $\begin{array}{l}\text { Median duration of mechanical ventilation before } \\
\text { weaning trial, day }\end{array}$ & $4(3-7)$ & $7(4-18)$ & 0.1 \\
\hline
\end{tabular}

Values are presented as or number (\%) unless otherwise indicated.

$\mathrm{IQR}$, interquartile range. 
Table 3. Comparison of physiologic and diaphragmatic values between patients with extubation success and failure

\begin{tabular}{|c|c|c|c|}
\hline Variable & Extubation success $(n=47)$ & Extubation failure $(n=13)$ & $p$ value \\
\hline \multicolumn{4}{|l|}{ Physiologic variable } \\
\hline Systolic blood pressure, mmHg & $122(113-128)$ & $125(117-130.8)$ & 0.534 \\
\hline Diastolic blood pressure, $\mathrm{mmHg}$ & $66(60-77)$ & $73.5(57 \cdot 3-85.8)$ & 0.309 \\
\hline Heart rate, beat/min & $83(71-90)$ & $89(74-102)$ & 0.279 \\
\hline Respiratory rate, breath/min & $18(15-20)$ & $19(15-21)$ & 0.570 \\
\hline Body temperature, ${ }^{\circ} \mathrm{C}$ & $36.5(36.4-36.6)$ & $36.45(36.3-36.5)$ & 0.256 \\
\hline PF ratio & $315.6(257.5-383.4)$ & $291.7(233.5-358.4)$ & 0.562 \\
\hline $\mathrm{PaCO}_{2}, \mathrm{mmHg}$ & $31(28-38)$ & $37(27 \cdot 5-43)$ & 0.351 \\
\hline \multicolumn{4}{|l|}{ Sonographic diaphragm parameter } \\
\hline Excursion, $\mathrm{cm}$ & $1.65(1.23-1.91)$ & $0.80\left(0.5^{1-1.1}\right)$ & $<0.001$ \\
\hline End-inspiration tdi, cm & $0.30(0.24-0.35)$ & $0.22(0.17-0.34)$ & 0.043 \\
\hline End-expiration tdi, cm & $0.21(0.18-0.24)$ & $0.19(0.15-0.26)$ & 0.434 \\
\hline$\Delta \operatorname{tdi} \%$ & $42.1(21.1-58.3)$ & $22.5(9.3-40)$ & 0.03 \\
\hline
\end{tabular}

Values are presented as median (interquartile range).

$\mathrm{PF}$, partial arterial oxygen pressure/fraction of inspired oxygen; $\mathrm{PaCO}_{2}$, arterial partial press of carbon dioxide; tdi, diaphragm thickness; $\Delta$ tdi\%, percent change of tdi between end-expiration and end-inspiration.

Table 4. Sonographic diaphragm parameters based on the type of respiratory failure

\begin{tabular}{|c|c|c|c|}
\hline Sonographic diaphragm parameter & Extubation success & Extubation failure & $p$ value \\
\hline Hypoxemic respiratory failure $(\mathrm{n}=37)$ & 32 & 5 & \\
\hline Excursion, cm & $1.69(1.06-1.96)$ & $0.98(0.84-1.75)$ & 0.248 \\
\hline End-inspiration tdi, cm & $0.28(0.20-0.35)$ & $0.32(0.20-0.42)$ & 0.714 \\
\hline End-expiration tdi, cm & $0.20(0.17-0.24)$ & $0.26(0.15-0.29)$ & 0.503 \\
\hline$\Delta \operatorname{tdi} \%$ & $39.4(16.9-55.6)$ & $35 \cdot 3(22.8-52.9)$ & 0.880 \\
\hline Hypercapnic respiratory failure $(n=23)$ & 15 & 8 & \\
\hline Excursion, $\mathrm{cm}$ & $1.65(1.23-1.90)$ & $0.69(0.32-1.03)$ & $<0.001$ \\
\hline End-inspiration tdi, cm & $0.31(0.27-0.39)$ & $0.21(0.16-0.24)$ & 0.001 \\
\hline End-expiration tdi, cm & $0.22(0.19-0.25)$ & $0.18(0.15-0.22)$ & 0.115 \\
\hline$\Delta$ tdi\% & $43.8(24.0-70.0)$ & $12.9(2.2-33.5)$ & 0.008 \\
\hline
\end{tabular}

Values are presented as interquartile range.

tdi, diaphragm thickness; $\Delta$ tdi\%, percent change of tdi between end-expiration and end-inspiration.

Table 5. Sensitivity, specificity, PPV, and NPV of sonographic indexes for extubation success

\begin{tabular}{lcccc}
\hline Variable & Sensitivity, \% & Specificity, \% & PPV, \% & NPV, \% \\
\hline Degree of excursion $\geq 1 \mathrm{~cm}$ & 80.9 & 69.2 & 90.5 & 50.0 \\
Degree of excursion $\geq 1.4 \mathrm{~cm}$ & 65.9 & 84.6 & 93.9 & 40.7 \\
$\Delta$ tdi $\geq 20 \%$ & 76.6 & 46.2 & 83.7 & 35.3 \\
$\Delta$ tdi $\geq 30 \%$ & 68.1 & 61.5 & 86.5 & 34.8 \\
\hline
\end{tabular}

PPV, positive predictive value; NPV, negative predictive value; tdi, diaphragm thickness; $\Delta$ tdi\%, percent change of tdi between end-expiration and end-inspiration. 


\section{DISCUSSION}

The present study shows that about one fifth of the critically ill patients who underwent extubation trial experienced extubation failure and those with extubation failure had less diaphragm excursion degree and $\Delta \mathrm{tdi} \%$. These findings suggest that diaphragm US may be a useful tool to predict successful extubation as previously reported. The excursion degree had higher area under the ROC curve than $\Delta$ tdi\% in the prediction of extubation success, which suggests the degree of diaphragm excursion may be more accurate than $\Delta$ tdi\% to predict successful extubation.

Extubation is an important process in the critically ill patients weaning from mechanical ventilation. Extubation is beneficial in terms of eliminating the work of breathing imposed by the endotracheal tube, lowering the risk for ventilator-associated pneumonia, enhancing patient comfort, and improving airway clearance by allowing an effective cough [10]. Extubation failure occurs in $10 \%$ to $30 \%$ of patients and is associated with poor prognosis $[1,11,12]$. The current study supports the previous findings that suggest an association between extubation failure and poor clinical outcomes. Therefore, proper extubation decision is crucial in improving the outcomes of critically ill patients. Some parameters are available to aid extubation decision, but they have several limitations [2]. Rapid shallow breathing index (RSBI) is used as a parameter deciding for the extubation timing during SBT, but its value is restricted to SBTs $[13,14]$.

The diaphragm is the principal respiratory muscle, and plays a key role in respiratory movement [3]. Diaphragm dysfunction predisposes patients to respiratory complications, can prolong the duration of mechanical ventilation and lead to weaning failure, resulting in poor prognosis in critically ill patients admitted to ICU [3]. Therefore, the evaluation of diaphragm function become essential to manage critically ill patients.

Demoule et al. [15] reported on diaphragm dysfunction upon ICU admission using twitch tracheal pressure in response to bilateral anterior magnetic phrenic nerve stimulation. They showed that $64 \%$ of patients had diaphragm dysfunction, which was associated with a poor prognosis [15]. There were also several methods to evaluate diaphragm function other than phrenic nerve stimulation [16]. US has been paid an attention to non- invasive, feasible, and accurate method of evaluating diaphragmatic function at the bedside [17]. Boon et al. [18] reported recently that US is a sensitive and specific tool for the diagnosis of neuromuscular diaphragm dysfunction. Two sonographic techniques have been used for diaphragmatic evaluation; one is the evaluation of diaphragm motion using M-mode US, and the other is assessment of diaphragm muscle thickening change during respiration $[5,8,19]$. Several studies have evaluated the diaphragm function using US in the critically ill patients $[6,7,20-23]$. Kim et al. [6] found that $29 \%$ of the patients with SBT had diaphragmatic dysfunction, which is defined as less than $1 \mathrm{~cm}$ in diaphragm excursion assessed through M-mode US. Additionally, they showed diaphragmatic dysfunction assessed via US was associated with weaning failure. They also suggested that the best diaphragmatic excursion cut-off values for predicting primary weaning failure may be at 14 and $12 \mathrm{~mm}$ for the right hemidiaphragm and left diaphragm, respectively and diaphragm US may be useful in identifying patients at high risk for weaning difficulty [6]. Valette et al. [20] investigated the prevalence of diaphragmatic dysfunction upon ICU admission using US. They found 10 patients $(2.2 \%)$ with diaphragm dysfunction who were admitted to the ICU for acute respiratory failure with poor prognosis. This study indicated that diaphragm dysfunction was not uncommon upon ICU admission in patients with acute respiratory failure. The change of tdi was also measured to predict weaning from mechanical ventilation [7,23]. DiNino et al. [7] investigated the association between $\Delta$ tdi\% at end-expiration and end-inspiration and extubation success during either SBTs or PS weaning trials. Their study showed that the combined sensitivity and specificity of $\Delta$ tdi $\% \geq 30 \%$ for extubation success were $88 \%$ and $71 \%$, respectively. The PPV and NPV were $91 \%$ and 63\%, respectively. They concluded that US measurement of diaphragm thickening in the zone of apposition may be useful to predict extubation success or failure during SBT or PS trials. The evaluation of diaphragm function assessed via US may be a useful index to predict weaning or extubation success.

Previous studies evaluated a single index of diaphragm US or compared to RSBI, but which sonographic indexes are better to predict extubation success remains to be elucidated. The current study showed that both aforementioned sonographic indexes are useful in the 
evaluation of diaphragm function to predict extubation success, which was supported by previous results $[6,7]$. Furthermore, the present study showed that diaphragm excursion degree may be a better index than $\Delta$ tdi\% to predict extubation success.

Several limitations of the present study should be addressed. First, selection bias cannot be excluded due to a small sample size and retrospective design, in which extrapolation of the findings to other settings must be done cautiously. Data were acquired in only an 8-month time period. Therefore, it seems feasible to perform a prospective study in a relatively short time period as well. We also acknowledge that currently several prospective papers have been published on this topic, making the significance of the current findings rather limited. Second, diaphragm excursion or thickness measurement was not immediately conducted before extubation. US timing may affect the diaphragm excursion or thickness change. Third, we only performed US on the right hemidiaphragm, not on the left hemidiaphragm, which may affect the extubation outcomes. Fourth, RSBI was not at the same time, so comparison of sonographic index and RSBI is not possible. In addition, we did not evaluate the diaphragm strength using magnetic phrenic nerve stimulation considered as currently the gold standard [15] and compare it with sonographic findings. Fifth, both medical and surgical critically ill patients were included and we did not limit the specific disease to evaluate diaphragm function through US. These factors might have an influence on the lower sensitivity or specificity of tdi than those of previous study.

In conclusion, US may be a valuable tool in the evaluation of diaphragm dysfunction and to predict extubation success. The diaphragm excursion degree obtained using US during SBT seems more accurate than the diaphragm thickening change to predict extubation success.

\section{KEY MESSAGE}

1. The diaphragm excursion degree was greater in patients with extubation success.

2. The diaphragm thickening change was greater in patients with extubation success.

3. Diaphragm excursion seems more accurate than the change of diaphragm thickness to predict extubation success.

\section{Conflict of interest}

No potential conflict of interest relevant to this article was reported.

\section{REFERENCES}

1. Epstein SK, Ciubotaru RL, Wong JB. Effect of failed extubation on the outcome of mechanical ventilation. Chest 1997;112:186-192.

2. Epstein SK. Decision to extubate. Intensive Care Med 2002;28:535-546.

3. McCool FD, Tzelepis GE. Dysfunction of the diaphragm. N Engl J Med 2012;366:932-942.

4. Heunks LM, Doorduin J, van der Hoeven JG. Monitoring and preventing diaphragm injury. Curr Opin Crit Care 2015;21:34-41.

5. Matamis D, Soilemezi E, Tsagourias M, et al. Sonographic evaluation of the diaphragm in critically ill patients: technique and clinical applications. Intensive Care Med 2013;39:801-810.

6. Kim WY, Suh HJ, Hong SB, Koh Y, Lim CM. Diaphragm dysfunction assessed by ultrasonography: influence on weaning from mechanical ventilation. Crit Care Med 2011;39:2627-2630.

7. DiNino E, Gartman EJ, Sethi JM, McCool FD. Diaphragm ultrasound as a predictor of successful extubation from mechanical ventilation. Thorax 2014;69:423-427.

8. Cohn D, Benditt JO, Eveloff S, McCool FD. Diaphragm thickening during inspiration. J Appl Physiol (1985) 1997;83:291-296.

9. Boles JM, Bion J, Connors A, et al. Weaning from mechanical ventilation. Eur Respir J 2007;29:1033-1056.

10. Artime CA, Hagberg CA. Tracheal extubation. Respir Care 2014;59:991-1002.

11. Thille AW, Harrois A, Schortgen F, Brun-Buisson C, Brochard L. Outcomes of extubation failure in medical intensive care unit patients. Crit Care Med 2011;39:26122618.

12. Frutos-Vivar F, Esteban A, Apezteguia C, et al. Outcome of reintubated patients after scheduled extubation. J Crit Care 2011;26:502-509.

13. Yang KL, Tobin MJ. A prospective study of indexes predicting the outcome of trials of weaning from mechanical ventilation. N Engl J Med 1991;324:1445-1450.

14. Lee KH, Hui KP, Chan TB, Tan WC, Lim TK. Rapid shallow breathing (frequency-tidal volume ratio) did not 
predict extubation outcome. Chest 1994;105:540-543.

15. Demoule A, Jung B, Prodanovic H, et al. Diaphragm dysfunction on admission to the intensive care unit: prevalence, risk factors, and prognostic impact: a prospective study. Am J Respir Crit Care Med 2013;188:213219.

16. Doorduin J, van Hees HW, van der Hoeven JG, Heunks LM. Monitoring of the respiratory muscles in the critically ill. Am J Respir Crit Care Med 2013;187:20-27.

17. Sarwal A, Walker FO, Cartwright MS. Neuromuscular ultrasound for evaluation of the diaphragm. Muscle Nerve 2013;47:319-329.

18. Boon AJ, Sekiguchi H, Harper CJ, et al. Sensitivity and specificity of diagnostic ultrasound in the diagnosis of phrenic neuropathy. Neurology 2014;83:1264-1270.

19. Boussuges A, Gole Y, Blanc P. Diaphragmatic motion studied by m-mode ultrasonography: methods, reproducibility, and normal values. Chest 2009;135:391-
400.

20. Valette X, Seguin A, Daubin C, et al. Diaphragmatic dysfunction at admission in intensive care unit: the value of diaphragmatic ultrasonography. Intensive Care Med 2015;41:557-559.

21. Mariani LF, Bedel J, Gros A, et al. Ultrasonography for screening and follow-up of diaphragmatic dysfunction in the ICU: a pilot study. J Intensive Care Med 2016;31:338343 .

22. Goligher EC, Laghi F, Detsky ME, et al. Measuring diaphragm thickness with ultrasound in mechanically ventilated patients: feasibility, reproducibility and validity. Intensive Care Med 2015;41:642-649.

23. Ferrari G, De Filippi G, Elia F, Panero F, Volpicelli G, Apra F. Diaphragm ultrasound as a new index of discontinuation from mechanical ventilation. Crit Ultrasound J 2014;6:8. 